(C) 2015 IEEE. Personal use of this material is permitted. Permission from IEEE must be obtained for all other uses, in any current or future media, including reprinting/republishing this material for advertising or promotional purposes, creating new collective works, for resale or redistribution to servers or lists, or reuse of any copyrighted component of this work in other works. 


\title{
Self-powered Wireless Sensor applied to Gear Diagnosis based on Acoustic Emission.
}

\author{
M. Delgado Prieto, Member, IEEE, D. Zurita, Student Member, IEEE, W. Wang, A. Machado, \\ J. A. Ortega, Member, IEEE, L. Romeral, Member, IEEE
}

\begin{abstract}
Advanced sensing strategies in the industrial sector are becoming a valued technological answer to increase the performance and competitiveness. The development of enhanced sensing solutions considering both technology and monitoring requirements is, nowadays, subject of concern in the industrial maintenance field. In this context, this work presents a novel selfpowered wireless sensor applied to condition monitoring of gears. The proposed sensor is based on a modular architecture, offering multipoint sensing, local wireless communication, multi-source energy harvesting and embedded diagnosis algorithm for mechanical fractures detection based on acoustic emission analysis. The developments are complemented by means of a remote management interface, from which the user can configure the functionalities of the sensors, visualize the network status as well as analyze the diagnosis evolution. The sensor performance, in terms of power consumption and fault detection, has been analyzed by means of experimental results.
\end{abstract}

Index Terms-Acoustic emission, health monitoring, intelligent sensor, power harvesting, remote monitoring, wireless sensor network.

\section{INTRODUCTION}

$\mathrm{D}$ URING the last years, wireless sensor are being adapted to a wide range of applications to provide a remote condition monitoring solution easily expandable [1-2]. The field of structural-health monitoring, for example, is gathering specific sensors and low-power strategies to be applied to buildings [3], and bridges [4], but also to aeronautical assembly inspections [5]. The healthcare applications have also received considerable attention to provide contextual information and alerting mechanisms about the people to be monitored [6]. Similarly, management and security needs have been approached in the agricultural and forest sector by means of automated irrigation structures or fire detection systems [78]. However, despite the great deal of sensors used for supervision purposes, the industrial sector shows a clear lack

This work was supported in part by the European seventh framework programme (FP7) for small medium enterprise under research project Mosycousis (reference number: 285848).

M. Delgado Prieto, D. Zurita, J. A. Ortega and L. Romeral are with the Motion Control and Industrial Applications, MCIA, Department of Electronic Engineering, Technical University of Catalonia, UPC, 08222 Terrassa, Spain (e-mail: miguel.delgado@mcia.upc.edu; daniel.zurita@mcia.upc.edu; juan.antonio.ortega@mcia.upc.edu; luis.romeral@mcia.upc.edu).

W. Wang is with the Tyndall National Institute, Dyke Parade, Cork, Ireland (e-mail: wensi.wang@tyndall.ie).

A. Machado is with the Energy Department, Fundació CTM Centre Tecnològic, 08242 Plaça de la Ciència 2, Manresa, Spain (e-mail: anderson.machado@ctm.com.es). of wireless sensor solutions for machinery monitoring. The massive introduction of wireless sensor networks in the industrial sector is restricted by two main challenges: first, the development of an automatic and autonomous sensor solution, capable of being integrated in a wireless network in order to centralize the information generated by a set of distributed sensors in a unique interface, and, second, the development of specific machinery monitoring algorithms embedded in the sensor [9-14].

Indeed, most of the available industrial sensors act like a data acquisition devices transmitting information to a sink node [15]. Instead of raw data transmission, on-sensor preprocessing is a better strategy to improve the overall performance [16-18]. However, the concept of embeddedsensing cannot be exploited if the system requires cables to access communication buses or if batteries have to be periodically replaced. In this regard, wireless technology and energy harvesting systems provide a wide range of solutions to become the sensor practically maintenance-free. Wireless local area networks can be easily implemented and transparent to the user, while multi-source energy harvester strategies are being proposed recently [22], based on the combination of vibration [19], thermoelectric [20] or solar/light [21] effects. Thus, the industrial monitoring system becomes highly scalable and flexible.

Dealing with industrial machinery failures, those related to the power transmission chain are the most common and concerning [23-24]. Among the different parts, the gearbox is found to be the most critical, since its downtime per failure is significant in comparison to other components [25]. Mechanical faults detection has been traditionally approached by means of vibration monitoring [26]. However, in order to reach the current condition monitoring requirements around earlier diagnosis and fault evolution tracking [27], the Acoustic Emission (AE), represents an advantageous option [28].

The originality of this work includes a complete selfpowered wireless sensor applied to mechanical fracture detection in gears by means of acoustic emission analysis. The developments include also a remote management interface in order to centralize the information from multiple sensors for management, visualization and interpretation purposes. Furthermore, this work introduces the $\mathrm{AE}$ technique to be embedded in the sensor processing layer for condition monitoring purposes. Although some approaches can be found in the literature [29-30], it should be noted that this is the first time that all these technologies, that is, AE sensing and 
processing, wireless communication and multi-source energy harvesting, converge in a unique sensor solution for industrial applications. Moreover, this work represents an important step to the introduction of wireless sensor devices and advanced sensing strategies to the development of industrial condition monitoring solutions.

The paper is organized as follows: sensor architecture and management is explained in Section II. The embedded diagnosis methodology is presented in Section III. The experimental results are explained in Section IV. Finally, this paper shows conclusion dissemination in Section V.

\section{SENSOR ARCHITECTURE AND MANAGEMENT}

The sensor unit has been envisaged as a compact device comprising of four interconnected modules, that is, the signal conditioning, the digital processing, the energy harvesting and the communications, as it is shown in Fig. 1.

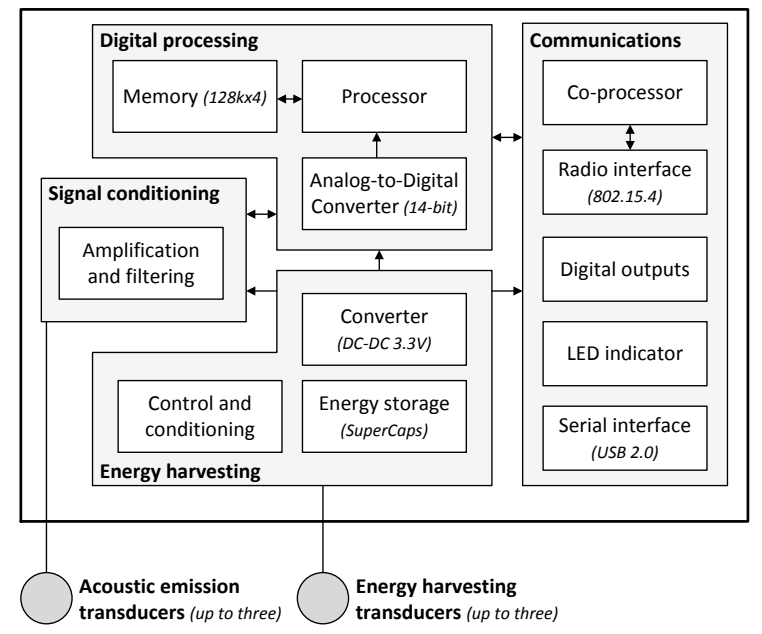

Fig. 1. Block diagram of the sensor unit: signal conditioning, digital processing, energy harvesting and communications. Three independent acoustic emission conditioning channels are considered. Three specific energy harvesters control and conditioning channels are included. Additionally, the sensor is featured with a general purpose light-emitting diode and three general purpose digital outputs.

An overview of the sensor modules is shown in Fig. 2. The modules are allocated in a prototype casing of $150 \mathrm{~mm} \times$ $120 \mathrm{~mm} \times 40 \mathrm{~mm}$. The ingress protection rating of the case is IP54 , making it suitable for most of industrial environments. The resulting packaged sensor unit is shown in Fig. 3.

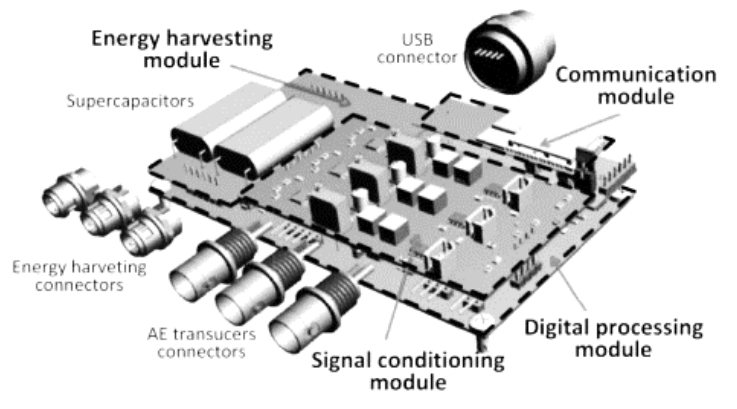

Fig. 2. Sensor unit illustration. Modules distribution and main connectors.
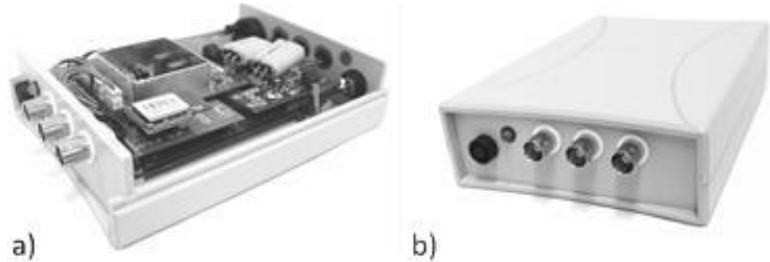

b)

Fig. 3. Sensor unit overview. a) Partial casing view. b) Complete casing view.

\section{A. Signal conditioning}

The signal conditioning represents the most critical module in terms of electronics design and implementation. The sensor is capable of managing up to three independent $\mathrm{AE}$ channels. Each channel is provided with low-noise-low-power amplifiers carrying out a band-pass filter around an $\mathrm{AE}$ spectral bandwidth of interest $(100 \mathrm{kHz}-400 \mathrm{kHz})$. Besides, this module allows a manual adjustment of the amplification ratio between $32 \mathrm{db}$ and $72 \mathrm{db}$. In order to increase the energy saving, the power supply distribution of the whole signal conditioning module is controlled by a solid-state switch commanded from a processor's digital output. The conditioned $\mathrm{AE}$ channels are connected to a 14-bits analog-todigital converter. A fixed sampling frequency of $2 \mathrm{MHz}$ during time windows of $10 \mathrm{msec}$. is applied, resulting in $280 \mathrm{Kbits}$ of data, and a spectral resolution of $100 \mathrm{~Hz}$.

\section{B. Digital processing}

The digital processing module is based on a digital processor, an ARM Cortex-M4, which implements the sensor management functions and the diagnosis algorithm. Internal memory is used to save the configuration parameters as well as intermedium signal processing results. The digital processor tasks are organized in four sequential stages: data acquisition, data processing, diagnosis algorithm and data transmission, as it is represented in Fig. 4. At the end of each stage, a low energy profile, the sleep mode, is applied to reduce the average energy consumption. The next stage will be executed after a sleep time, $t_{\text {sleep }}$, and the verification of a certain state of charge of the energy storage unit, $S o C_{t h}$, otherwise the sleep mode will remain during an additional $t_{\text {sleep }}$.

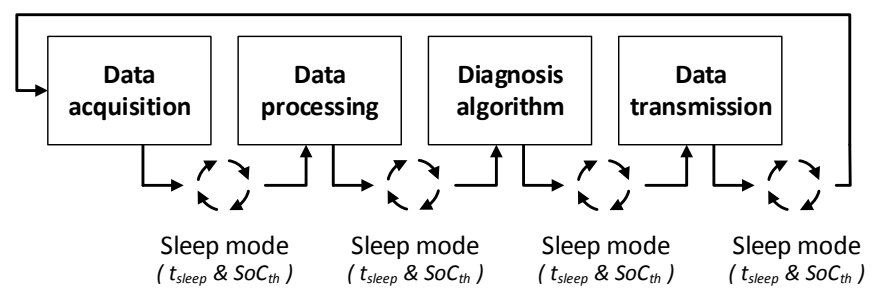

Fig. 4. Operating cycle carried out by the digital processor. Previously to the execution of each task, the sensor unit remains under sleep mode during a sleep time, $t_{\text {sleep }}$. Then, the state of charge of the battery, $S o C_{t h}$, is checked in order to allow the execution of the next task.

\section{Energy harvesting}

The energy harvesting module has been designed to avoid battery package dependency, which implies a costly maintenance requirement. Dealing with energy harvesting, a 
multi-source approach has been adopted in order to allow flexibility and improve the energy availability. Thus, the proposed energy harvesting module allows up to three different energy sources connections. First, the vibration energy harvester, which allows the conversion of machinery residual vibration in electric energy by means of the piezoelectric effect. A Perpetuum FSH module for $50 \mathrm{~Hz}$ mains region with a power output up to $20 \mathrm{~mW}$ has been adopted. Second, the thermoelectric energy generator that takes advantage of thermal gradients trough the Seebeck effect. A customized harvester has been designed based on an array of $255 \mathrm{Bi}_{2} \mathrm{Te}_{3}$ thermoelectric-material prepared into a $P$ and $N$ types of $1 \mathrm{~mm}^{3}$ thermos-elements. A matrix arrangement supported with ceramic substrates results in a 50 $\mathrm{mm}^{2}$ of harvester able to provide up to several milliWatts at temperature differences around $75^{\circ} \mathrm{C}$. And finally, third, a low voltage indoor photovoltaic harvester based on four Sanyo AM1815 amorphous silicon solar cells. In case of significant variations of the operating conditions (frequency/amplitude in vibration or temperature gradient), the consideration of such harvester is proposed. The cumulative photovoltaic energy is sufficient for self-powered the sensor as a secondary source able to maintain hundreds of microWatts as a constant power delivering ratio.

The energy harvesting module has been designed to support an initial specification of $2 \mathrm{~mW}$ of sensor unit power consumption considering the whole set of functionalities, which corresponds to $125 \mathrm{~mJ}$ of energy supply per minute. Taking into account such consumption ratio, the energy storage unit has been designed by means of two $5 \mathrm{~F}$ supercapacitors. This capacity offers and autonomy of several hours under an energy harvesting drop scenario. The supercapacitors have been selected also considering a low equivalent series resistance. This features allows a better performance during the energy transients of the sensor, i.e. transitions from sleep mode (seconds of microWatts), to a wireless transmission mode (milliseconds of miliWatts).

The module is completed by means of input power conditioning circuits between each harvester and the storage device. Then, the harvesting module includes rectifier and DC/DC converter for electromagnetic (vibration) energy harvester, ultra-low voltage DC/DC converter for thermoelectric generator, and a maximum power point device fixed at $80 \%$ for indoor photovoltaic cells. An additional load switch and an output voltage regulator (buck/boost converter), are used to supply $3.3 \mathrm{~V}$ to the rest of the electronics. The load switch and the enable pin of the buck/boost converter are controlled by a digital battery pin $\left(V_{B A T T_{-} O K}\right)$. Thus, the buck/boost converter starts up when is fully operational and the cold start issue of buck/boost converter is avoided.

\section{Communications}

The selection of the wireless personal area network technology has been carried out taking into consideration the application requirements. The 802.15.4 based technology represents a good tradeoff between low power consumption (energy saving modes), range of operation (tens of meters), and baud rate (kilobits per second). Although multiple standards are available, the ZigBee technology represents one of the most mature one, with a good penetration in the market. Thus, the implemented radio interface is based on a NXP JN5148 Zigbee wireless coprocessor. The additional wired communication mode is solved by a 2.0 USB interface. These two communication channels allow the interaction with the user through the Management Interface (MI).

\section{E. Management interface}

The management interface (MI), collects and presents the information in regard with the presence of mechanical degradation in the point/s under test, and also about the operating status (i.e. battery level), of each sensor unit.

During the regular operation mode, Fig. 5(a), each sensor unit (SU), sends trough a wireless connection the corresponding information to a management interface, installed in a remote host computer. In order to minimize communication loads (power consumption), a simplex star network topology has been defined, in which the data transfer is carried out non-periodically (under energy availability constraints), from the SU to the host computer. Thus, the proposed monitoring wireless network allows an autonomous and continuous on-site diagnosis. In order to manage automatically the network (i.e. association process with sensor units), a wireless hub, wired linked to the management interface host computer, is included. The wireless hub is seen, from the host computer, as a serial port from which data frames are received containing the corresponding fields, such as sensor unit identifier, diagnosis outcome and sensor unit energy status. Complementary, the SU can be wired connected to the MI host computer. In the wired mode, Fig. 5(b), the SU functionalities and diagnosis parameters can be configured, as well as massive AE raw data can be downloaded. a)
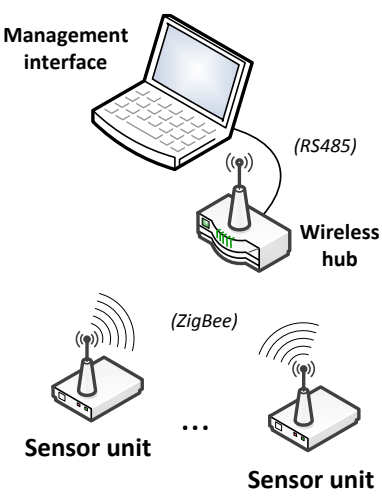

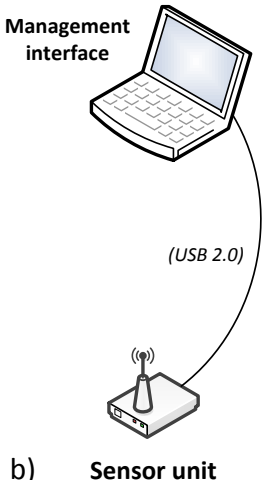

b)
Fig. 5. Communication modes of the sensor unit. a) Wireless mode for regular operation. Multiple sensor units can be connected directly to the management interface throughout a wireless hub. b) Wired mode for sensor configuration or acoustic emission raw data download from the management interface.

The architecture of the management interface is modular in order to isolate different functionalities and allow an easy update. Indeed, the MI functionalities have been grouped in four modules: interfaces, analysis, data manager and communications. 
A communication module of the MI has been implemented as library using .NET platform and specific serial port handling from Windows operating system. The wireless operation has been categorized in four scenarios as follows:

--OutOfBox, a WSU is connected for first time to the network (the WSU must be registered into the MI and calibrated).

--PowerSOS, the harvesting power supplier level of a SU reach a specified threshold $h p s_{t h}$.

--Alive, a WSU wakes up from a no-energy status (available energy below $h p s_{t h}$ ).

--Data, regular data frame containing information regarding diagnosis outcome and SU status.

An example of wireless communication dialogue between a SU and the MI is shown in Fig. 6(a), where the four kinds of considered frames are represented. In case of wired communication, Fig. 6(b), the actions are selected by the user: configuration of the SU functionalities, massive AE raw data download, diagnosis parameters configuration or $\mathrm{SU}$ functional tests.
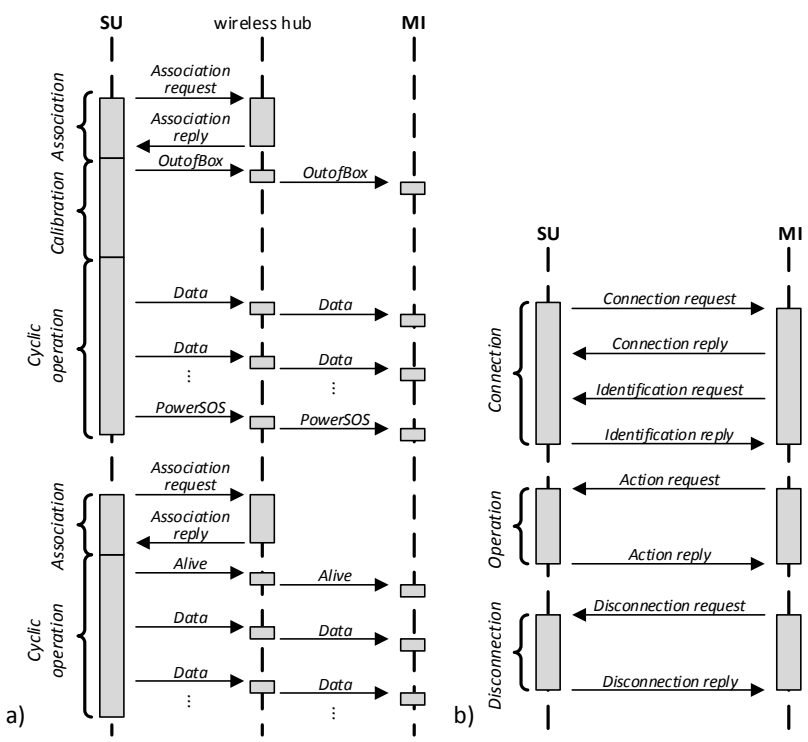

Fig. 6. Communication dialogues between the sensor unit and the management interface. a) Wireless communication between a SU and the MI throughout the wireless hub. First, after the initial connection of the SU to a power source (battery level > hps $s_{t h}$ ), and OutofBox frame is sent. Following the default procedure, the SU carries out its calibration. Later, Data frames are sent non-periodically to the MI. Previous to a critical energy level, the SU sends a PowerSOS frame. In case of an energy decrease below $h p s_{t h}$, the SU will turn off and when there is availability will restart the association procedure to the network, Alive frame sending and Data frames transmission. b) Wired communication between a WSU and the RMI. The connection and disconnection corresponds to automatic procedures requested by the WSU and the RMI respectively. The operations are executed sequentially in a requestreply mode.

A data manager module handles the database by means of .NET platform and MySQL database as backend. The analysis library module, based on MATLAB runtime engine, contains the diagnosis procedure to be applied over the download $\mathrm{AE}$ raw data. Finally, the user interface module has been designed as graphical application using .NET platform and Windows Forms and PostgreSQL connector as it is shown in Fig. 7.

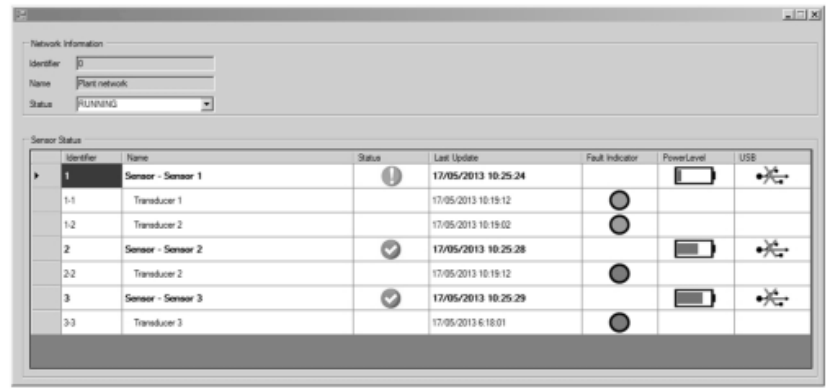

Fig. 7. Management interface main panel view. Three sensor units are registered in the network, the first one, sensor 1, with two associated $\mathrm{AE}$ transducers and a low energy warning, the second and the third ones with one associated $\mathrm{AE}$ transducer each other and no warning message.

\section{Diagnosis Methodology}

The proposed on-site data processing and diagnosis algorithm aims to detect fracture evidence in the point of measurement under mechanical stress. Thus, in order to develop the proposed methodology, mechanical fatigue tests over individual F114 steel gears has been carried out by means of specific testing machinery. The objective of the experiments is to characterize $\mathrm{AE}$ signal signatures under different mechanical fracture stages.

Gears are subjected to fatigue cycles until the elastic module of the material is reached and a fracture appears. It should be noticed that the tests were not carried to final fracture of gear teeth. The tests were stopped once cracks were evidenced macroscopically. A $30 \mathrm{kN}$ sinusoidal load of 10-12 $\mathrm{Hz}$ is applied to the gear, $\mathrm{AE}$ is acquired periodically each 30 seconds with a sampling frequency of $6 \mathrm{MHZ}$. The samples are acquired by means of AMSY-5 Vallen system AE resonant sensors. The fracture produced in the gear at the end of the experiment is shown in Fig. 8.

Generally, during the fracture process, the acoustic activity (number of AE events that overpass an amplitude threshold) of a material can be classified into three different phases: crack initiation, crack incubation, and crack propagation [31-32]. Fig. 9 shows the evolution of the AE activity in regard with the fatigue applied to the sample, where the three fracture stages can be easily identified. This fact means that there exist three different $\mathrm{AE}$ patterns to be analyzed in order to characterize each degradation stage.
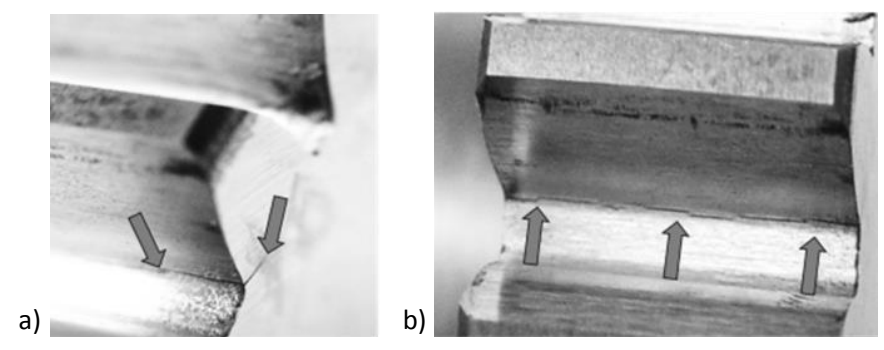

Fig. 8. Detail of resulting cracks generated within the gear under the mechanical fatigue tests. (a) Lateral tooth crack propagation. (b) Transversal tooth crack propagation.

The three characteristics signatures extracted from the experiment are shown in Fig. 10. The AE activity increases throughout the mechanical fracture evolution, as well as the presence of $\mathrm{AE}$ at higher frequency bands. The frequency 
spectrum of AE signals in stage 1 presents a maximum around $100 \mathrm{kHz}$, Fig. 10(a). In stage 2, AE signals have higher amplitudes than in stage 1 and the frequency reaches $200-250$ $\mathrm{kHz}$, Fig 10(b). In stage 3, the amplitude of AE signals increases considerably, and frequency peaks extend over a higher range of values between 100 and $800 \mathrm{kHz}$, Fig. 10(c).

In basis of the existent literature [33-34], and the results of the experiments, it can be concluded that there is a characteristic AE pattern which permits to discern faults generated in fatigue tests. This pattern is based in the fact that there is a shift on the spectral content towards higher frequencies directly related with the mechanical degradation stage.

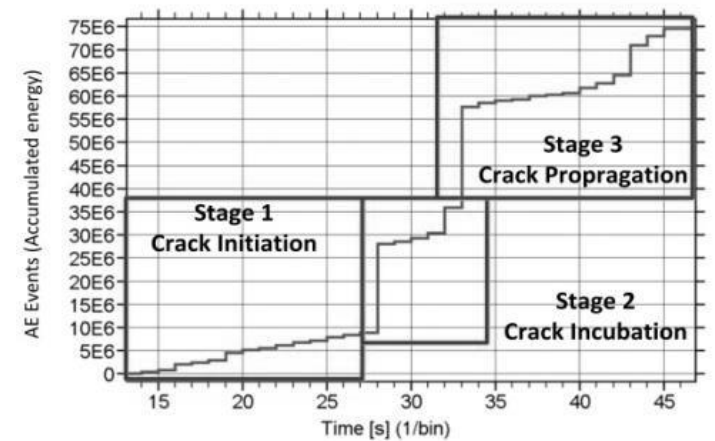

Fig. 9. AE results in terms of AE energy vs duration of the test. Sample loaded to $\mathrm{F}_{\max }=30 \mathrm{kN}$ and $\mathrm{N}=20100$ cycles. Three stages are identified during the experiment, (i) initiation, (ii) incubation, and (iii) propagation.

Thus, the proposed diagnosis methodology estimates the mechanical fracture degradation by analysing the ratio of $\mathrm{AE}$ energy among three different frequency bands. However, as it has been mentioned, due to the limitations of the sensor, the predefined $10 \mathrm{msec}$. time of acquired $\mathrm{AE}$ signal implies the consideration of short and non-periodic segments of the corresponding mechanism working cycle. These eventual diagnosis outcomes, here called indicators of mechanical fracture, $F_{\text {deg }}$, must be understood as probabilistic measurements. For this reason, the analysis of an $F_{d e g}$ historic is considered to ensure the stability of the final diagnosis, resulting in a proposed numerical value, $M_{d}$, which represents the estimated mechanical fracture degradation percentage. As shown in Fig. 11, the diagnosis methodology proceeds as follows:

1) The acquired $\mathrm{AE}$ signal is band-pass filtered over the first spectral band, $f_{b I}$ (from $100 \mathrm{kHz}$ to $150 \mathrm{kHz}$ ). This band is considered the baseband for further comparatives. Tthe
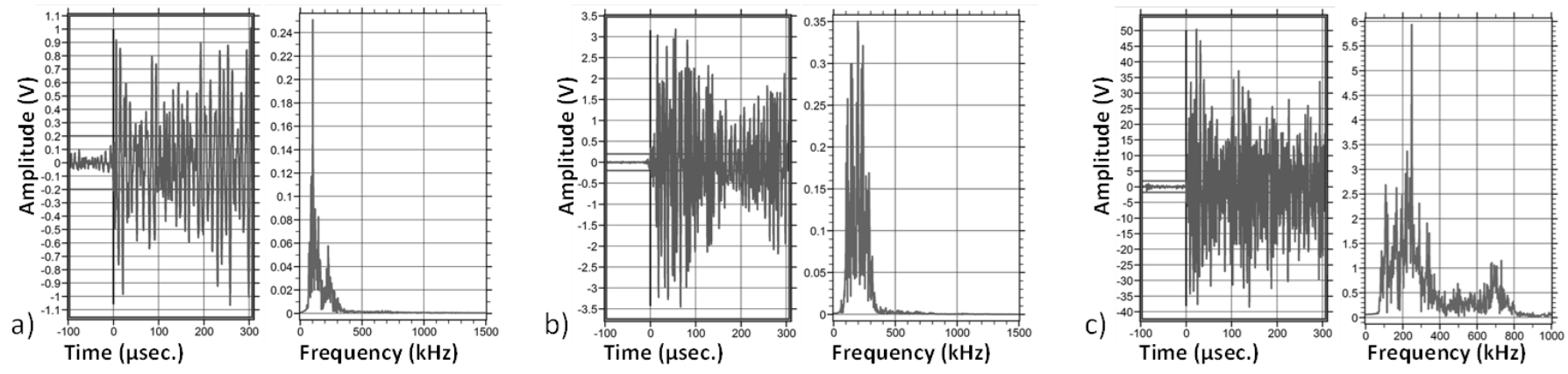

Fig. 10. Examples of characteristic AE signals obtained from the mechanical fatigue test in different degradation stages. The vertical reference marks the same instant of time. The horizontal reference shows the same threshold value. a) Temporal and spectral AE signal obtained from degradation stage 1 - crack initiation. b) Temporal and spectral AE signal obtained from stage 2 - crack incubation. c) Temporal and spectral AE signal obtained during stage 3 - crack propagation. 


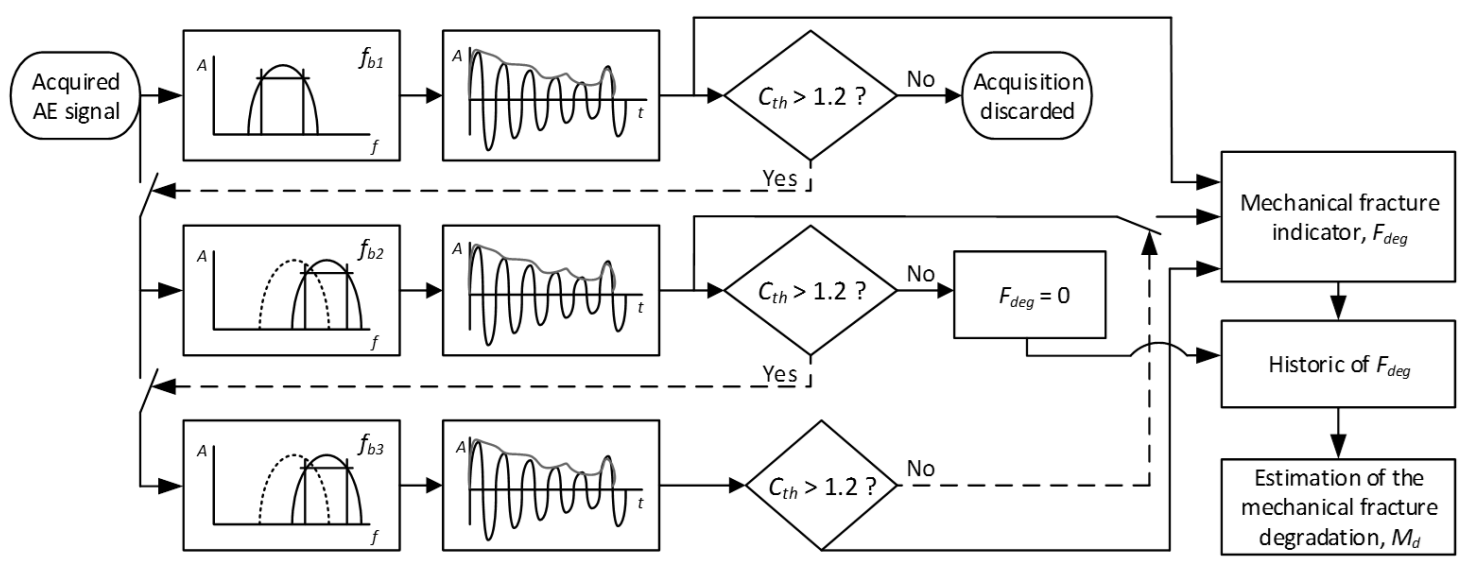

Fig. 11. Proposed diagnosis methodology. Three spectral bands, $f b_{1}, f b_{2}$ and $f b_{3}$ are considered over the acquired AE signal in order to analyze their contents and estimate the mechanical fracture indicator, $F_{d e g}$, and the final mechanical fracture degradation diagnosis outcome, $M_{d}$.

6) Then, the mechanical fracture evaluation corresponds to the relation of the baseband, $r m s_{f b l}$, and the rms of the highest band with $C_{t h}>1.2, r m s_{h b}$, as it is shown in (3).

$$
F_{d e g}=\frac{r m s_{h b}}{r m s_{f b 1}} \cdot \frac{W_{\text {factor }}}{F_{\text {max }}} \cdot 100
$$

In order to provide quantitative information in regard with the mechanical fracture, a maximum fault level, $F_{\max }$, is defined. This parameter corresponds to the maximum expected relation between the $r m s_{f b l}$ and $r m s_{f b 3}$. This parameter is set to 3 corresponding to the maximum energy relation found in the fatigue experiments for the crack propagation phase.

7) The resulting $F_{d e g}$ is saved in a historical database formed by the $N$ last values, where $N$ is set at 100 in order to analyze 100 acquisition of $10 \mathrm{~ms}$, which has been proven to be sufficient in mechanical elements such as the gears used in the experiments. The $M_{d}$ is obtained from the historic assessment by means of (4).

$$
M_{d}=\frac{1}{N} \sum_{i=1}^{N} F_{\operatorname{deg}_{i}}
$$

Finally, after the assessment of multiple experimental results, it is proposed a numerical interpretation of the results formed by four regions in regard with the value of $M_{d}$ such as:

$$
\begin{array}{cc}
\text { Healthy } & 0 \leq M_{d}<20 \\
\text { Initial degradation } & 20 \leq M_{d}<50 \\
\text { Significant degradation } & 50 \leq M_{d}<85 \\
\text { Generalized degradation } & 85 \leq M_{d}<100
\end{array}
$$

As it has been aforesaid such diagnosis procedure is carried out by the WSU during the regular wireless mode operation. In this mode $10 \mathrm{msec}$. of $\mathrm{AE}$ signal is acquired nonperiodically, under energy availability constraints. In case of wired mode operation, continuous long period of AE signal (i.e. several seconds), can be acquired and stored in the RMI host-computer and, then, the $M_{d}$ value can be likewise estimated by the same diagnosis procedure. The wired mode allows an instantaneous mechanical degradation assessment once the $F_{\text {deg }}$ historic is completed.

\section{EXPERIMENTAL RESULTS}

In order to evaluate the performance of the proposed sensor unit, two experimental analyses have been carried out. The first analysis is focused on the fault detection capabilities, while the second analysis lies on the power consumption and energy harvesting capabilities.

\section{A. Mechanical fracture degradation}

Taking into consideration that it is not feasible to induce controlled mechanical degradations over operating industrial machinery, the validation of the system is done in the laboratory on an electromechanical test bench, reproducing mechanical subsystems in an industrial plant as shown in Fig. 12. The electromechanical system consists of permanent magnet synchronous machine acting as a drive connected to a 1:1 rated gearbox. A commercial AE transducer, Vallen Systeme VS900-M, offering a $50 \mathrm{kHz}$ to $500 \mathrm{kHz}$ spectral band, is located in contact with the gearbox chassis. Three different rotating speeds conditions have been considered: 150,250 and $450 \mathrm{rpm}$. Two gears have been used to carry out the diagnosis validation, a healthy gear, $H_{G}$, and a gear with high severity failure ( 8 fractures at different teeth bases between $1 \mathrm{~mm}$ and $5 \mathrm{~mm}), F_{G}$.

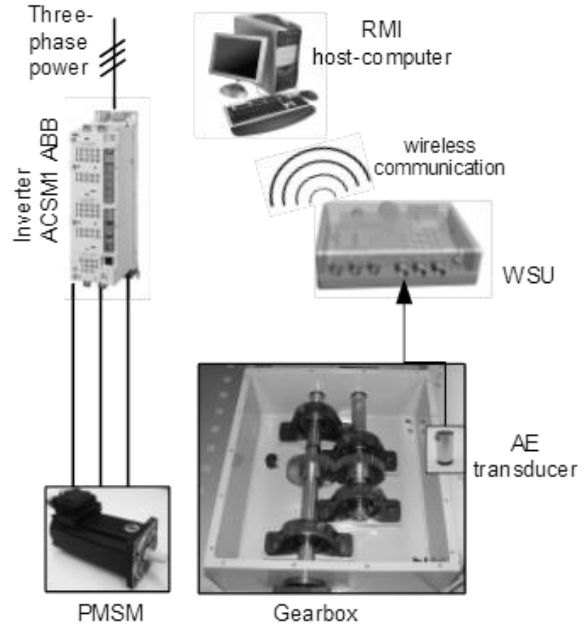

Fig. 12. Experimental setup for mechanical fracture detection in gears. 
The sampling frequency is fixed at $2 \mathrm{MHz}$. A total amount of 20 ksamples are acquired, as a result, each acquisition corresponds to $10 \mathrm{msec}$ of $\mathrm{AE}$ signal. The same characterized gear samples, used during the fatigue tests, are mounted in the gearbox. It is shown in Fig. 13 and Fig. 14 the resulting AE signal processing following the proposed diagnosis methodology for both $H_{G}$ and $F_{G}$ cases.

a)

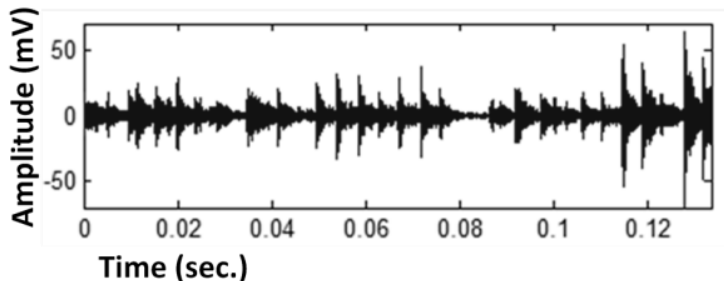

b)

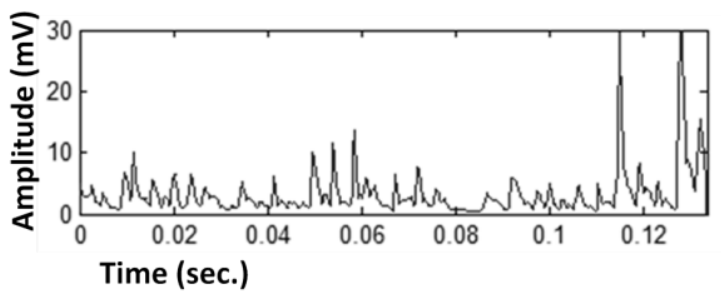

c)

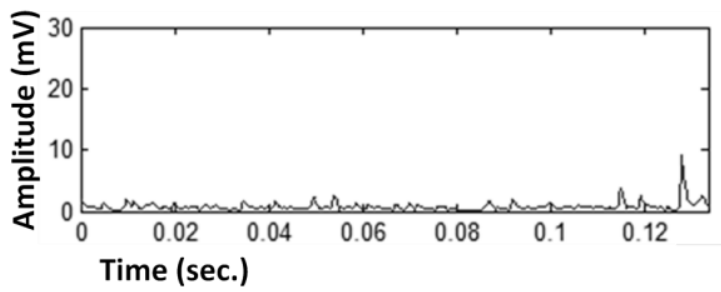

d)

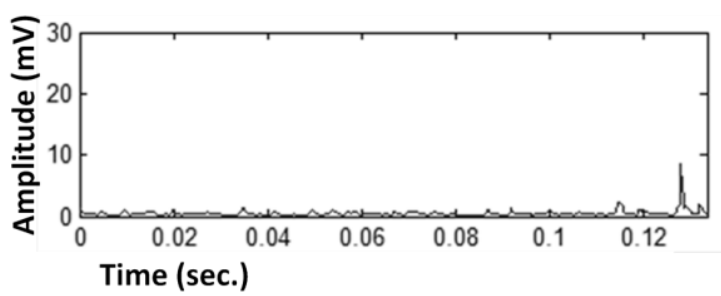

Fig. 13. Filtering process over an acoustic emission signal corresponding to one revolution at 450rpm in a healthy gearbox based test bench. a) Temporal acoustic emission signal acquired. b) Resulting temporal acoustic emission signal after a band-pass filter $\left(f b_{l}, 100 \mathrm{kHz}-150 \mathrm{kHz}\right)$ and time envelope. c) Resulting temporal acoustic emission signal after a band-pass filter $\left(f b_{2}\right.$, $150 \mathrm{kHz}-200 \mathrm{kHz}$ ) and time envelope. d) Resulting temporal acoustic emission signal after a band-pass filter $\left(f b_{3}, 200 \mathrm{kHz}-250 \mathrm{kHz}\right)$ and time envelope.

The estimation of the rms over the resulting AE signal at $f b_{1}, f b_{2}$ and $f b_{3}$ allows obtaining the indicator of mechanical fracture, $F_{\text {deg }}$. This value has been obtained over $100 \mathrm{AE}$ acquired signals during the test bench operation in order to complete the $F_{\text {deg }}$ historic length. Thus, the results of the evaluation of $M d$ can be seen in the next Table I. The results shown that under a healthy gear condition, the $M_{d}$ value is around $13-15 \%$, and when the gear is under faulty condition $(8$ degraded teeth in our case), the indicator increases up to 70 $73 \%$ indicating clearly the presence of degradation in the gear. The $M d$ values in Table I correspond to an average calculation among the $M d$ values obtained during 10 repetitions of each of the six experiments. It should be noticed that the $M d$ values in Table I exhibit a small deviation of $\pm 2.5 \%$ max. when different operating speeds are considered, which represents a good stability of the proposed diagnosis methodology. These results reveal that the analysis of the spectral content of the $\mathrm{AE}$ events shown properly the corresponding gear condition. The nature of the identified $\mathrm{AE}$ patterns related with the fracture implies that the estimation of common time-based condition indicators such as rms, peak to peak or kurtosis, are not be capable to characterize the considered degradations.

a)

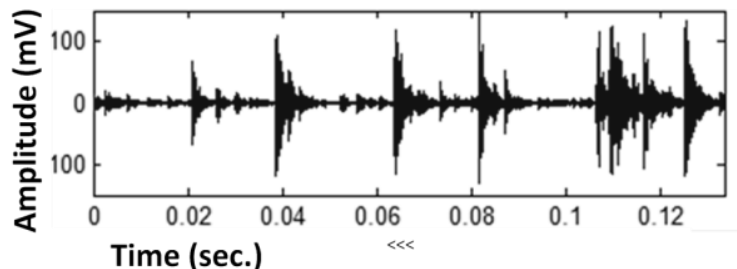

b)

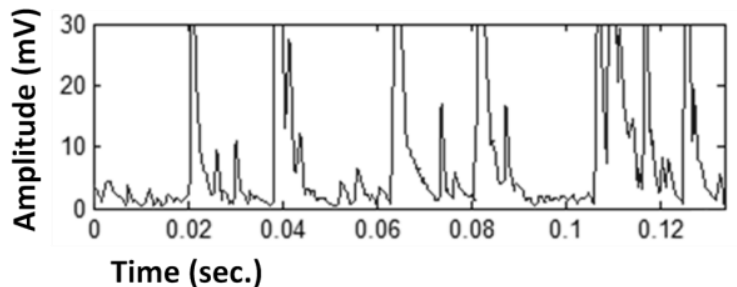

c)

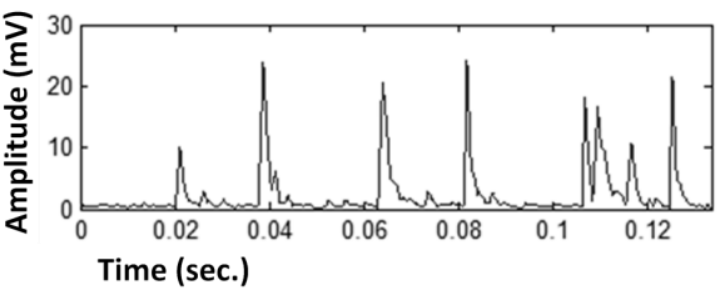

d)

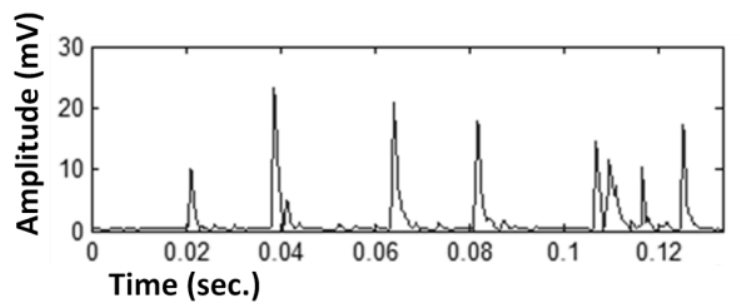

Fig. 14. Filtering process over an acoustic emission signal corresponding to one revolution at 450rpm in a faulty gearbox based test bench. a) Temporal acoustic emission signal. b) Resulting temporal acoustic emission signal after a band-pass filter $\left(f b_{1}, 100 \mathrm{kHz}-150 \mathrm{kHz}\right)$ and time envelope. c) Resulting temporal acoustic emission signal after a band-pass filter $\left(f b_{2}, 150 \mathrm{kHz}-\right.$ $200 \mathrm{kHz}$ ) and time envelope. d) Resulting temporal acoustic emission signal after a band-pass filter $\left(f b_{3}, 200 \mathrm{kHz}-250 \mathrm{kHz}\right)$ and time envelope.

TABLE I

RESULTING $M_{d}$ UNDER THREE OPERATING CONDITIONS. MEAN VALUE, STANDARD DEVIATION AND 95\% CONFIDENCE INTERVAL CONSIDERING 10 REPETITIONS OF THE EXPERIMENTS

\begin{tabular}{ccccc}
\hline & & $150 \mathrm{rpm}$ & $250 \mathrm{rpm}$ & $450 \mathrm{rpm}$ \\
\hline Healthy & Mean & 13.99 & 13.53 & 15.59 \\
\cline { 2 - 5 } gear & $S t d$. & 0.37 & 0.48 & 0.53 \\
\cline { 2 - 5 }$H_{G}$ & $C I_{a t 95 \%}$ & {$[13.76-14.21]$} & {$[13.23-13.83]$} & {$[15.26-15.92]$} \\
\hline Faulty & Mean & 70.82 & 71.68 & 73.32 \\
\cline { 2 - 5 } gear & $S t d$. & 2.11 & 1.79 & 2.29 \\
\cline { 2 - 5 }$F_{G}$ & $C I_{a t 95 \%}$ & {$[69.51-72.13]$} & {$[70.57-72.79]$} & {$[71.90-74.74]$} \\
\hline
\end{tabular}


Numerical time-domain features are related with a measure of $\mathrm{AE}$ intensity, which can lead to false positive outcomes. Moreover, such AE intensity measures require the characterization of all the operating scenarios in order to identify diagnosis thresholds for each of them. The resulting analysis shown in Table I can be compared with the analysis of the experimental $\mathrm{AE}$ data by means of time-based indicators shown in Fig. 15.
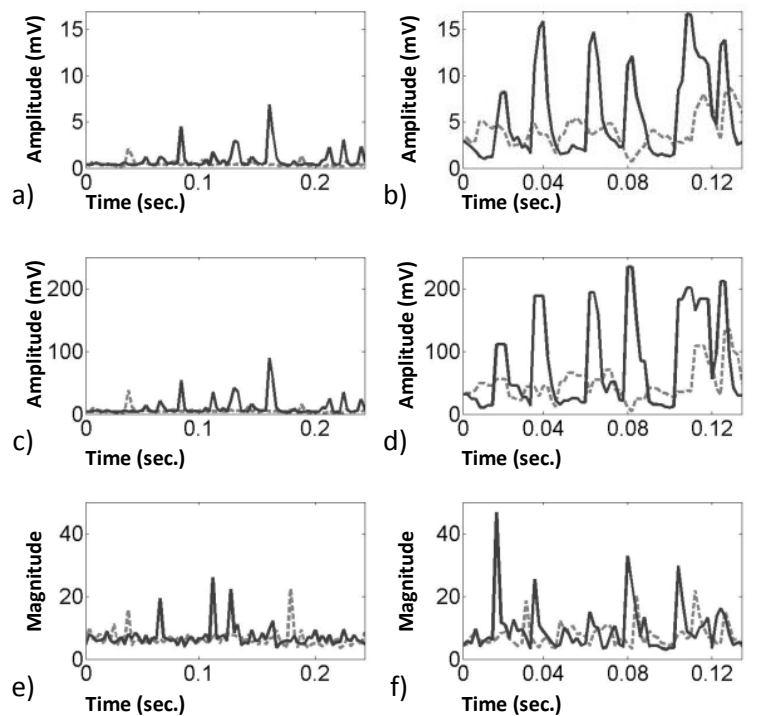

Fig. 15. Statistical time-domain features. Dotted line: AE signal under a healthy gearbox scenario, Continuous line: $\mathrm{AE}$ signal under a faulty gearbox scenario. a) rms estimation at $250 \mathrm{rpm}$. b) rms estimation at $450 \mathrm{rpm}$. c) peak to peak estimation at $250 \mathrm{rpm}$. d) peak to peak estimation at 450rpm. e) kurtosis estimation at 250rpm. f) kurtosis estimation at 450rpm.

\section{B. Sensor power consumption}

A low power consumption profile represents an important objective during the design of the SU. Power measurements over the SU operation show consumptions between 80$180 \mathrm{~mW}$ power while executing main tasks, and $50 \mu \mathrm{W}$ power during the sleep mode. The consumption details are summarized in Table II. The power consumption is estimated by measuring the required load current and voltage delivered by the supercapacitors.

TABLE II

SU POWER CONSUMPTION DETAILS

\begin{tabular}{cccc} 
& $\begin{array}{c}\text { Power } \\
{[\mathrm{mW}]}\end{array}$ & $\begin{array}{c}\text { Time } \\
{[\text { sec.] }}\end{array}$ & $\begin{array}{c}\text { Energy } \\
{[\mathrm{mJ}]}\end{array}$ \\
\hline Sleep mode, $T_{S}$ & 0.047 & 60.00 & 2.820 \\
\hline $\begin{array}{c}\text { Data acquisition } \\
\text { (three channels })\end{array}$ & 188.1 & 0.020 & 3.760 \\
\hline Data processing & 76.26 & 0.920 & 70.16 \\
\hline Diagnosis algorithm & 86.16 & 0.210 & 18.09 \\
\hline Data transmission & 61.38 & 0.220 & 13.50 \\
\hline \hline Total (@ $T_{s}=$ Imin. $)$ & 1.76 & 61.37 & 108.3 \\
\hline Total (@ $T_{s}=3$ min. $)$ & 0.62 & 181.3 & 113.9 \\
\hline Total (@ $T_{s}=$ 10min.) & 0.22 & 601.3 & 133.7
\end{tabular}

On average, the active mode time is approximately 1.37 seconds, followed by the sleep mode time, $T_{S}$, set at 1,3 and
10 minutes in the tests. Depending on the $T_{S}$, the average power consumption ranges from $0.22 \mathrm{~mW}$ to $1.76 \mathrm{~mW}$.

In order to verify the WSU autonomy, available ambient energy sources has been tested. The mechanical vibration energy and surface temperatures on various positions of an standard $40 \mathrm{~kW}$ air compressor unit have been measured to study the available energy. In this characterization, $0.025 \mathrm{~g}$ to $0.05 \mathrm{~g}$ vibration has been detected in addition to the $60-70{ }^{\circ} \mathrm{C}$ surface temperature on the rotary screw air compressor (near the air outlet). Based on the characterization, thermoelectric energy harvesting is proposed in this work. The supercapacitor voltage and charge time are measured to calculate the harvested power and energy. The average harvested power $P_{\text {avg }}$ can be derived from the super-capacitor energy $E_{S C}$ and charge time $T_{\text {chrg }}$ as (6)

$$
P_{a v g}=\frac{E_{s c}}{T_{c h r g}}=\frac{C_{s c} \cdot\left(V_{\text {final }}^{2}-V_{\text {init }}^{2}\right)}{2 \cdot T_{c h r g}}
$$

where $C_{S C}$ is the super-capacitor capacitance, $V_{\text {final }}$ and $V_{\text {init }}$ are the final voltage and initial voltage of the supercapacitor. The results are shown in Fig. 16.

The harvested thermoelectric energy is calculated based on the voltage change on the super-capacitor at $1.01 \mathrm{~J}$ within 300 seconds. The average harvested power of thermal energy harvester is calculated at $3.37 \mathrm{~mW}$. The charge experiment was also conducted with different capacitors, the harvested power remains the same value.

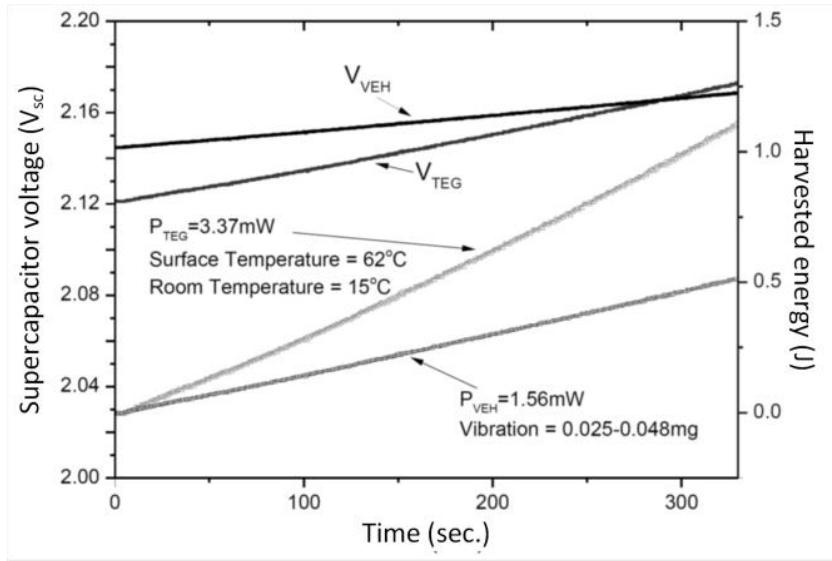

Fig. 16. Vibration and thermal harvesters (VEH and TEG respectively) during supercapacitor charging experiments. Supercapacitor voltage $\left(V_{V E H}\right.$ and $\left.V_{T E G}\right)$, and harvested power $\left(P_{V E H}\right.$ and $\left.P_{T E G}\right)$.

The vibration of the air compressor tested in this experiment varies significantly due to its different operation modes, 25$48 \mathrm{mg}$ magnitude at $49.3-49.7 \mathrm{~Hz}$ frequency is measured. The harvested energy is measured at $470 \mathrm{~mJ}$ in 300 seconds.

The harvested vibrational power is $1.56 \mathrm{~mW}$ on average during the charging process. Whilst the air compressor is operational, the total energy harvested power is calculated at $4.93 \mathrm{~mW}$. The harvested power is sufficient to power the WSU operating at $T_{S}=1$ minute $(1.76 \mathrm{~mW}$ in Table I). Based on the duty cycle power consumption data of the WSU, the proposed multi-source energy harvester supplies enough power to decrease the $T_{S}$ until 20 seconds. 


\section{CONCLUSIONS}

This paper introduces a novel self-powered wireless sensor device applied to mechanical fracture detection in gears by means of acoustic emission analysis.

The proposed solution integrates four different key technologies in a unique sensor device, that is, up to three acoustic emission channels, wired/wireless communications, multi-source energy harvesting, and embedded diagnosis algorithm. Multiple sensor devices can be easily managed by means of the proposed management interface, which, from a host computer, allows the visualization of all the information sent by the distributed set of sensors in the plant.

The proposed embedded diagnosis algorithm has been designed by means of specific mechanical fracture characterization procedures over gear samples. The proposed diagnosis methodology is based on the analysis of the spectral content of the acoustic emission signal, since the different mechanical fracture stages analyzed during the experiments show characteristic spectral contents.

The performance of the proposed sensor solution in terms of power autonomy and diagnosis results has been tested.

In regard with the power consumption, the sensor solution requires less than $110 \mathrm{~mJ}$ of energy at a ratio of one diagnosis cycle per minute. This energy harvesting requirement has been proved to be realistic by means of an energy harvesting experiments carried out over an industrial air compressor.

The proposed embedded diagnosis algorithm has been validated over a gear based electromechanical test bench. The results show a very good performance during the identification. Moreover, the resulting diagnosis outcome is very stable in front of speed conditions variations.

It should be noted that this sensor solution can be applied in whatever electromechanical structure where gears are present. The potential of the diagnosis algorithm, together with the sensor possibilities allowing three different points of test, has the capability to extract the information coming from the mechanical fracture itself that from other machine vibration sources.

\section{REFERENCES}

[1] I. F. Akyildiz and M. C. Vuran, "Factors Influencing WSN Design," in Wireless Sensor Networks, 1st ed. Ed. United Kingdom: John Wiley and Sons Ltd., 2010, pp. 37-49.

[2] J. Lu, H. Okada, T. Itoh, T. Harada and R. Maeda, "Toward the World Smallest Wireless Sensor Nodes With Ultralow Power Consumption," IEEE Sensors J., vol. 14, no. 6, pp. 2035-2041, Jun. 2014.

[3] T. Torfs, T. Sterken, S. Brebels, J. Santana, R. van den Hoven, V. Spiering, N. Bertsch, D. Trapani and D. Zonta, "Low Power Wireless Sensor Network for Building Monitoring," IEEE Sensors J., vol. 13, no. 3, pp. 909-915, Mar. 2013.

[4] M. J. Whelan, M. V. Gangone and K. D. Janoyan, "Highway bridge assessment using an adaptive real-time wireless sensor network," IEEE Sensors J., vol. 9, no. 11, pp. 1405-1413, Nov. 2009.

[5] T. Becker, M. Kluge, J. Schalk, K. Tiplady, C. Paget, U. Hilleringmann and T. Otterpohl, "Autonomous Sensor Nodes for Aircraft Structural Health Monitoring," IEEE Sensors J., vol. 9, no. 11, pp. 1589-1595, Nov. 2009.

[6] H. Alemdar and C. Ersoy, "Wireless sensor networks for healthcare: A survey," Computer Networks, vol. 54, no. 15, pp. 2688-2710, Oct. 2010.

[7] J. Gutierrez, J. F. Villa-Medina, A. Nieto-Garibay and M. A. PortaGandara, "Automated Irrigation System Using a Wireless Sensor Network and GPRS Module," IEEE Trans. Instrum. Meas., vol. 63, no. 1, pp. 166-176, Jan. 2014.
[8] B. C. Arrue, A. Ollero and J. R. Matinez de Dios, "An intelligent system for false alarm reduction in infrared forest-fire detection," IEEE Intell. Syst., vol. 15, no. 3, pp. 64-73, Mar. 2000.

[9] G. Zhao, "Wireless Sensor Network for Industrial Process Monitoring and Control: A Survey", Network Protocols and Algorithms, vol. 3, no. 1, pp. 46-63, Feb. 2011.

[10] L. A. Gupta and D. Peroulis, "Wireless Temperature Sensor for Condition Monitoring of Bearings Operating Through Thick Metal Plates," IEEE Sensors J., vol. 13, no. 6, pp. 2292-2298, Jun. 2013.

[11] C. H. R. Martins, P. R. Aguiar, A. Frech and E. C. Bianchi, "Tool Condition Monitoring of Single-Point Dresser Using Acoustic Emission and Neural Networks Models," IEEE Trans. Instrumentation and Measurement, vol. 63, no. 3, pp. 667-679, Mar. 2014.

[12] A. Ukil, M. Zlatanski and M. Hochlehnert, "Monitoring of HV Generator Circuit Breaker Contact Ablation Based on Acoustic Emission," IEEE Trans. Instrumentation and Measurement, vol. 62, no. 10, pp. 2683-2693, Oct. 2013

[13] J.-H. Zhou, C. K. Pang, Z.-W. Zhong and F. L. Lewis, "Tool Wear Monitoring Using Acoustic Emissions by Dominant-Feature Identification," IEEE Trans. Instrumentation and Measurement, vol. 60, no. 2, pp. 547-559, Feb. 2011.

[14] I. Bua-Nunez, J. E. Posada-Roman, J. Rubio-Serrano and J. A: GarciaSouto, "Instrumentation System for Location of Partial Discharges Using Acoustic Detection With Piezoelectric Transducers and Optical Fiber Sensors," IEEE Trans. Instrumentation and Measurement, vol. 63, no. 5, pp. 1002-1013, May 2014.

[15] B. Aygün and V. C. Gungor, "Wireless sensor networks for structure health monitoring: recent advances and future research directions," Sensor Review, vol.31, no.3, pp.261-276, 2011.

[16] L. Hou and N. W. Bergmann, "Novel Industrial Wireless Sensor Networks for Machine Condition Monitoring and Fault Diagnosis," IEEE Trans. Instrumentation and Measurement, vol.61, no.10, pp.27872798, Oct. 2012.

[17] W. Y. Toh, Y. K. Tan, W. S. Koh and L. Siek, L., "Autonomous Wearable Sensor Nodes With Flexible Energy Harvesting," IEEE Sensors J., vol. 14, no. 7, pp. 2299-2306, Jul. 2014.

[18] J. Hu, J. Januar and C. Zhao, "Vibration energy harvesting based on integrated piezoelectric components operating in different modes," IEEE Trans. Ultrasonics, Ferroelectrics, and Frequency Control, vol.57, no.2, pp.386-394, Feb. 2010.

[19] L. Hou and N. W. Bergmann, "Novel Industrial Wireless Sensor Networks for Machine Condition Monitoring and Fault Diagnosis," IEEE Trans. on Instrumentation and Measurement, vol. 61, no. 10, pp. 2787-2798, Oct. 2012.

[20] J. Kim and C. Kim, "A DC-DC Boost Converter With VariationTolerant MPPT Technique and Efficient ZCS Circuit for Thermoelectric Energy Harvesting Applications," IEEE Trans. Power Electronics, vol.28, no.8, pp.3827-3833, Aug. 2013.

[21] A. Decker, "Solar energy harvesting for autonomous field devices," IET Wireless Sensor Systems, vol.4, no.1, pp.1-8, Mar. 2014.

[22] A. S. Weddell, M. Magno, G. V. Merrett, D. Brunelli, B. Al-Hashimi and L. Benini, "A survey of multi-source energy harvesting systems," in Proc. Design, Automation \& Test in Europe Conference \& Exhibition, , pp.905-908, 18-22 Mar. 2013.

[23] M. Ognjanovic and M. Benur, "Experimental research for robust design of power transmission components," Meccanica, vol.46, no.4, pp.699710, Aug. 2011.

[24] L. Ruoyu and D. He, "Rotational Machine Health Monitoring and Fault Detection Using EMD-Based Acoustic Emission Feature Quantification," IEEE Trans. Instrumentation and Measurement, vol. 61, no. 4, pp. 990-1001, Apr. 2012.

[25] I. Yesilyurt, "The application of the conditional moments analysis to gearbox fault detection - a comparative study using the spectrogram and scalogram," NDT \& E International, vol.37, no.4, pp.309-320, Jun. 2004.

[26] C. K. Tan, P. Irving and D. Mba, "A comparative experimental study on the diagnostic and prognostic capabilities of acoustics emission, vibration and spectrometric oil analysis for spur gears," Mechanical Systems and Signal Processing, vol.21, no.1, pp.208-233, Jan. 2007.

[27] D. Galar, A. Thaduri, M. Catelani and L. Ciani, "Context awareness for maintenance decision making: A diagnosis and prognosis approach," Measurement, Vol. 67, pp. 137-150, May 2015.

[28] C. U. Grosse and M. Ohtsu, "Acoustic Emission Testing," 2nd ed., Ed. Germany:Springer, 2010. 
[29] A. Ledeczi, T. Hay, P. Volgyesi, D.-R. Hay, A. Nadas and S. Jayaraman, "Wireless Acoustic Emission Sensor Network for Structural Monitoring," IEEE Sensors Journal, vol. 9, no. 11, pp. 1370-1377, Nov. 2009.

[30] I. Chilibon, M. Mogildea and G. Mogildea, "Wireless Acoustic Emission Sensor Device with Microcontroller," Procedia Engineering, vol. 47, pp. 829-832, 2012.

[31] Shi Z., Jarzynski J., Hurlebaus S., Jacobs LJ. Characterization of acoustic emission signals from fatigue fracture. Proceedings of the Institution of Mechanical Engineers 214-9 (2000) 1141-1149.

[32] K. Tanaka, "Fatigue Crack Propagation," in Comprehensive Structural Integrity, ed. I. Milne, R.O. Ritchie and B. Karihaloo, Pergamon, Ed: Oxford, pp.95-127, 2003.

[33] D. Mba and R. B. K. N. Rao, "Development of Acoustic Emission Technology for Condition Monitoring and Diagnosis of Rotating Machines: Bearings, Pumps, Gearboxes, Engines, and Rotating Structures," The Shock and Vibration Digest, vol. 38, no.1, pp.3-16, 2006.

[34] A. Choudhury and N. Tandon, "Application of acoustic emission technique for the detection of defects in rolling element bearings," Tribology International, vol.33, no.1, pp.39-45, Jan. 2000. 